\title{
High Postoperative Tacrolimus Variability Predisposes to Early Pancreas Graft Loss
}

\author{
Mihnea loan Ionescu' ${ }^{1}$ Avinash Sewpaul, Rodrigo Figueiredo, Jakub Chmelo, Steven Alan White, \\ Derek Michael Manas, Colin Hugh Wilson
}

'Department of HPB and Transplant Surgery, Newcastle upon Tyne Hospitals NHS Foundation Trust, Freeman Hospital, Newcastle upon Tyne, NE7 7DN , United Kingdom

\section{ABSTRACT}

Background: The therapeutic index for tacrolimus (Tac) is relatively narrow and can vary postoperatively in pancreas transplantation (PancTx) recipients secondary to various factors. We hypothesised that intra-patient Tac variability (\%Coefficient of Variance; Tac \%CoV) after PancTx would influence early patient outcomes.

Methods: 45 pancTx recipients in our centre between 2009-2014 were retrospectively reviewed. The \%CoV was calculated using their inpatient Tac levels [(standard deviation Tac trough level/mean Tac trough level) $x$ 100]. Patients were then separated into 2 groups, of HIGH and LOW variability, depending on their relationship to the median and outcomes were compared.

Results: Tac \%CoV ranged between $19.02 \%$ and $114.9 \%$ with a mean of $46.95 \%$ (median value $45 \%$ ). Patients with a value $>45 \%$ (HIGH) had a decreased 1 year survival of $58 \%$ vs. $83 \%(p=0.026)$. Cold ischaemia time (HR 1.263, $p=0.019)$ and an episode of rejection whilst an in-patient (HR 3.656, $p=0.049)$ are predictors of graft loss.

Conclusions: Tac \%CoV is significantly greater in PancTx recipients when compared with kidney transplant recipients. Even in the era of Campath induction, it is possible that more grafts are being lost to rejection related complications than previously thought. Further studies are required before definitive conclusions can be drawn.

Key words: tacrolimus variability, pancreas transplantation, graft loss, acute rejection

\section{INTRODUCTION}

The introduction of calcineurin inhibitors dramatically improved the outcomes for solid organ transplant recipients in the 1980's and 1990's. Tacrolimus is the cornerstone of most immunosuppressive drug maintenance regimens in the modern era; however, therapeutic index for tacrolimus (Tac) is relatively narrow and can vary postoperatively in PancTx transplant patients secondary to drugs, diabetic gastroparesis and changes in bowel motility. Variable blood drug concentrations have been shown to be a risk factor for rejection and long-term allograft nephropathy in kidney transplantation (1). Patients on tacrolimus are at
Corresponding author: Mihnea loan Ionescu, MD 11 Cloister Garth, South Gosforth Newcastle upon Tyne, NE7 7LW United Kingdom

Email: mihnea.ioan@gmail.com mihnea.ionescu@nuth.nhs.uk
Received: 15.05.2017 Accepted: 02.06.2017 
risk for adverse events related to both excessive and insufficient immunosuppression. Since then evidence from other organ transplants is steadily emerging that confirms the usefulness of tacrolimus intra-patient variability as a predictor of clinical outcomes including long term graft function in transplant recipients (2-5). However, there is a paucity of data in PancTx regarding the influence of early tacrolimus levels on outcomes in PancTx.

We hypothesised that intra-patient Tac variability (\%Coefficient of Variance; Tac \%CoV) would influence early patient outcomes after PancTx despite our use of alemtuzumab depleting antibody induction.

\section{METHODS}

\section{Patients}

Retrospectively collected data from patients undergoing a solid organ pancreatic transplant from 01 January 2009 - 31 December 2014 were examined. Patient medical records, a computer database and hospital computer electronic patient records identified 45 patients. Of the 45 patients identified, we excluded 1 patient as the graft was lost very early. We examined variables such as cold ischaemia time, donor and recipient demographic data, HLA mismatches, episodes of rejection as an inpatient as well as 30 day mortality. Our primary endpoint was graft survival.

\section{Surgery, immunosuppression and post-operative antibiotic prophylaxis}

The majority of our patients in this study received a simultaneous pancreas and kidney transplant with enteric drainage to the jejunum; pancreas alone grafts (PAK, PTA) and where there were doubts about duodenal vascularity were exocrine drained via the bladder. All patients received alemtuzumab (Lemtrada ${ }^{\circledR}$; Genzyme Therapeutics Ltd, Oxford, UK) $30 \mathrm{mg}$ subcutaneously injected (SC) and methylprednisolone 500 $\mathrm{mg}$ intraoperatively. Postoperatively recipients received tacrolimus (Prograf ${ }^{\oplus}$; Astellas Pharma Ltd, Chertsey, UK) $\mathrm{BD}(0.05 \mathrm{mg} / \mathrm{kg} \mathrm{PO}$ target trough whole blood concentration between 8 and $12 \mu \mathrm{g} / \mathrm{L}$ ), mycophenolate mofetil (MMF; Cellcept ${ }^{\circledR}$; Roche Pharma AG, Grenzach Wyhlen, Germany) $500 \mathrm{mg}$ BD and a further dose of alemtuzumab $30 \mathrm{mg}$ SC. The antimicrobial prophylaxis consists of Micafungin (Mycamine ${ }^{\circledR}$; Astellas Pharma Europe, Leiden, Netherlands) and Meropenem (Hospira UK Limited, Maidenhead, UK) following anaesthetic induction. Antimicrobials were continued for 3 days alongside oral gut decontamination: neomycin (Alliance Healthcare (Distribution) Ltd., London, UK) and Colistin (Colomycin ${ }^{\circledast}$,
Forest Laboratories UK Ltd., Dartford, UK) for enteric drained pancreas transplants.

\section{CMV and HSV prophylaxis}

Valganciclovir (Valcyte ${ }^{\circledR}$, Roche Products Ltd., West Sussex, UK) was administered for CMV mismatch and recipient positive patients with the dose adjusted according to estimated glomerular filtration rate as determined by the Cockcroft-Gault equation for at least 100 days. For $D$ - to $R$ - transplant oral acyclovir was preferred for viral prophylaxis.

\section{Anticoagulation}

In our institution thromboprophylaxis has evolved over the study period. Now prophylaxis is guided by thromboelastography (TEG). Dextran 40 , as well as unfractionated and fractionated heparin are all used to maintain a hypocoagulable TEG (Coagulation Index less than 0).

\section{Diagnosis of rejection}

Pancreas allograft function is commonly monitored by a number of parameters including serum amylase, lipase, glycated haemoglobin ( $\mathrm{HbA} 1 \mathrm{c})$, or, if bladder drained, urinary amylase. In simultaneous transplants the kidney was biopsied as a surrogate for rejection if there was clinical suspicion or alternatively empirical bolus steroid therapy initiated if the biochemical parameters were suggestive of rejection.

\section{Intra-patient tacrolimus variability (tacrolimus variability)}

We used the following formula to calculate the \% intra-patient tacrolimus variability:

\section{$\%$ Coefficient of Variance of Tacrolimus = (standard deviation tacrolimus trough level/mean tacrolimus trough level) $x 100$.}

The trough tacrolimus level of each patient was collected whilst the patient remained in hospital. The available results were subsequently dichotomised into low and high variability groups.

\section{Statistical analysis}

The independent samples $t$-test was used to compare mean age between subgroups. The MannWhitney $U$ test was applied to compare non-parametric ordinal data, and the chi-squared test or Fisher's exact test was applied for analysis of categorical variables. Graft survival between the 2 subgroups was analysed using Kaplan Meir survival curves. A Cox proportionalhazards analysis was performed, but not a multivariate 
analysis as the datasets were considered too small for meaningful analysis.

\section{RESULTS}

A total of 44 patients were identified and included in the analysis. Tac \%CoV ranged between $19.02 \%$ and $114.9 \%$ with a mean of $46.95 \%$ (median value of $45 \%$ ). Patients were allocated to high or low tacrolimus variability subgroups as determined by the median cutoff value of $45 \%$.

Median follow-up was 15 months (range 6-73 months). Table 1 depicts the general patient characteristics at time of surgery, median major HLA mismatches, cold ischaemia time, mean donor age, the type of graft the patients received, the 30 day mortality and the number of episodes of rejection (as an inpatient) in both subgroups.

The patients were fairly well matched in each subgroup as seen in figs. 1 and 2 .

There were 4 suspected cases of rejection. Of these 2 were in patients with SPKs and 2 in patients with a pancreas alone graft. Only one of the renal grafts in an SPK recipient was biopsied on suspicion of rejection.

In order to visualize the clinical importance of the observed effect of intra-patient variability of tacrolimus on graft failure, a Kaplan-Meier curve was constructed for patients with a low and a high variability for tacrolimus clearance. Patients with a value $>45 \%$ (HIGH) had a 1 yr survival of $58 \%$ vs. $83 \%$ ( $p=0.026$ ) (fig. 3).
Table 2 summarises the results of the univariate analysis. In our model we found cold ischaemia time (hazard ratio 1.263, $\mathrm{p}=0.019$ ), an episode of rejection whilst an in-patient (hazard ratio $3.656, p=0.049$ ) and tacrolimus variability to be predictors of graft loss on univariate analysis (hazard ratio 1.021, $\mathrm{p}=0.034$ ).

\section{DISCUSSION}

There is a paucity of data in PancTx relating exposure of recipients to tacrolimus and graft outcomes. In this study, we have found a significant correlation between the in-patient tacrolimus variability and the end point of graft failure. Although "between-patient" variability has been studied extensively, there are hardly any studies in which the importance of "intra-patient" variability of immunosuppressive drugs for clinical outcome has been investigated in PancTx. Patients with high variability in clearance have more fluctuations in the measured tacrolimus concentrations. As a result, the blood concentrations will more often be outside the therapeutic window causing either toxicity (in the case of overexposure) or the potential for immune recognition and rejection (in the case of underexposure). This may explain the poor outcomes associated with high withinpatient variability.

We have only looked at the tacrolimus variability of patients whilst they were an inpatient in the immediate postoperative period following their PancTx as the doses and changes were regular, well documents and within

Table 1. Patient characteristics and variables in both subgroups

\begin{tabular}{|c|c|c|c|}
\hline Variable & $\begin{array}{l}\text { Low tacrolimus variability } \\
\qquad(\mathrm{n}=22)\end{array}$ & $\begin{array}{l}\text { High tacrolimus variability } \\
\qquad(\mathrm{n}=22)\end{array}$ & $p$ value \\
\hline Mean Age (range) & $44.59(28.8-57.04)$ & $43.13(29-56)$ & $p=0.524$ \\
\hline $\begin{array}{l}\text { Sex } \\
\text { Male } \\
\text { Female }\end{array}$ & $\begin{array}{l}9 \\
13\end{array}$ & $\begin{array}{c}18 \\
4\end{array}$ & $p=0.012$ \\
\hline Number of HLA mismatches (median) & 4 & 4 & \\
\hline Cold ischaemia time (CIT) (hrs) & 11.44 & 12.91 & $p=0.146$ \\
\hline Mean donor age (range) & $40.5(9-55)$ & $40.1(17-60)$ & $p=0.9$ \\
\hline $\begin{array}{l}\text { Type of graft } \\
\text { SPK } \\
\text { PAK } \\
\text { Pancreas alone }\end{array}$ & $\begin{array}{c}13 \\
3 \\
6 \\
\end{array}$ & $\begin{array}{c}18 \\
0 \\
4\end{array}$ & $p=0.9$ \\
\hline 30 day mortality rate & $0 \%$ & $4.54 \%$ & $\begin{array}{c}p=0.312\left(x^{2}=1.01\right. \\
\text { Chi squared test) }\end{array}$ \\
\hline Number of in-patient episodes of rejection & 1 & 3 & $p=0.6069$ \\
\hline Tac \%CoV (mean) & 34.96 & 58.95 & $p<0.001$ \\
\hline
\end{tabular}


Figure 1.

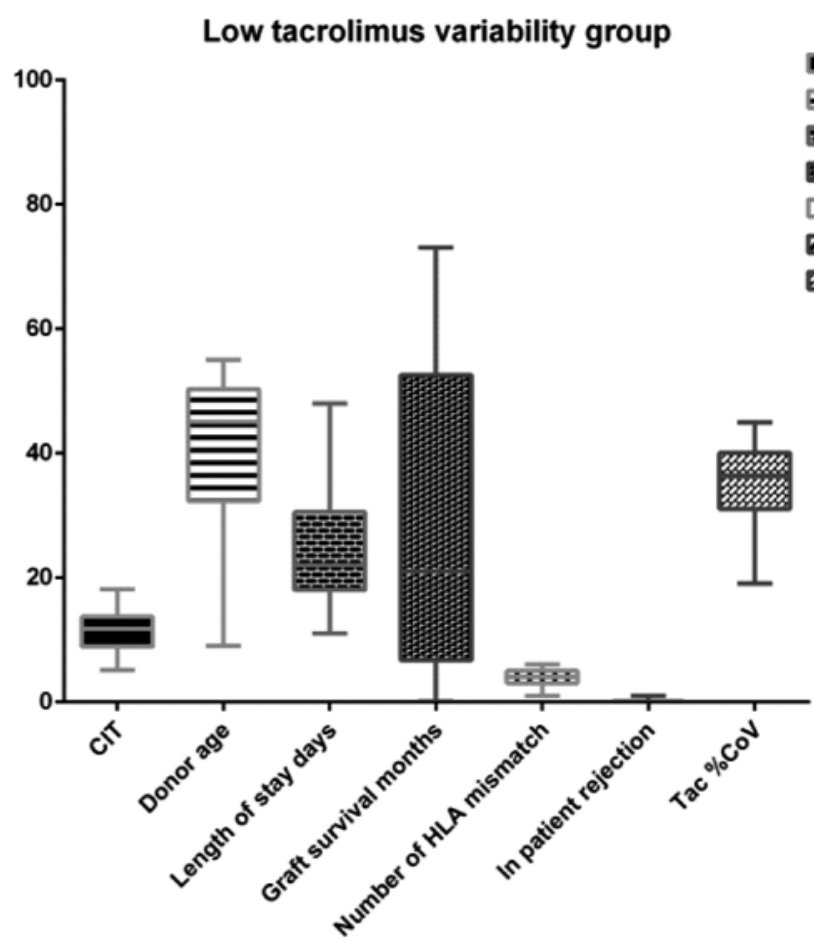

CIT E Donor age

포 Length of stay days

Graft survival months

W0 Number of HLA mismatch

[D In patient rejection

389 Tac \%CoV

Figure 2.

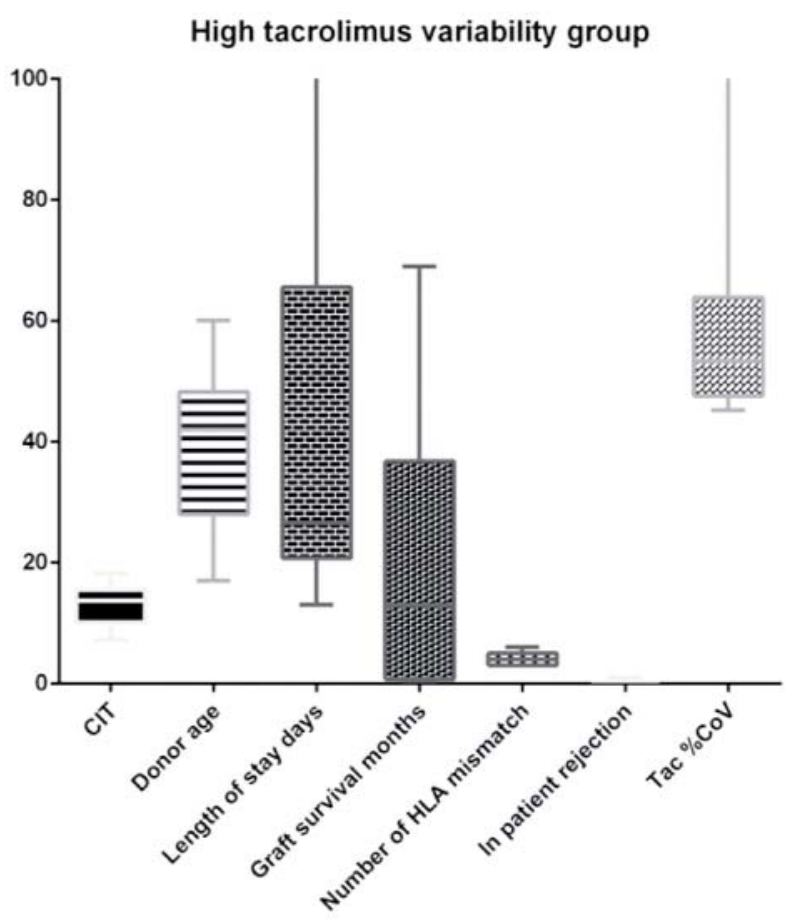

- CIT

- Donor age

Length of stay days

Graft survival months

UII Number of HLA mismatch

"I In patient rejection

एac $\% \mathrm{CoV}$ our own institution. Many of our patients are returned to the referring centres after hospital discharge and variations in outcome could then be related to geographical factors as well. The choice of high and low tacrolimus variability groups (45.08\%) could be criticised as arbitrary, but we felt with this small dataset and the precedent of a previous study in the literature [Borra et al (6)] that such an approach had the merit of simplicity. In the Borra study their cut-off value to differentiate between high and low variability was $14.9 \%$. Their 


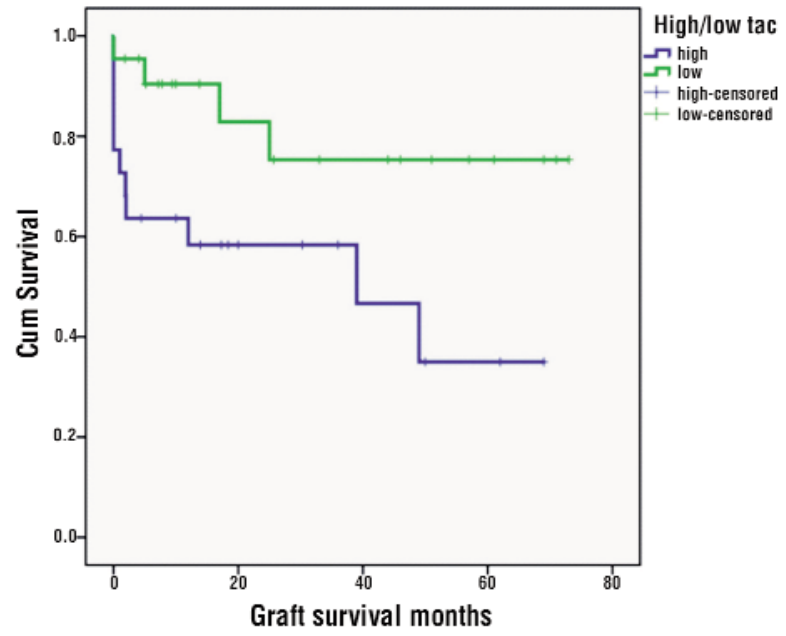

Figure 3 - Kaplan Meier Survival curves for high and low in patient tacrolimus variability. The mean graft survival for the high and low tacrolimus groups were 19.83 and $\mathbf{2 8 . 9 5}$ months respectively (Log-rank test $p=0.026$ )

cohort was in-patients who had received kidney transplants, but seen at follow-up between 6-12 months when their tacrolimus levels were measured.

In the literature various statistical approaches have been used to determine pharmacokinetic variance. Sapir-Pichhadze et al used the standard deviation of tacrolimus levels as their interpretation of tacrolimus variability (7) as did Pollock-Barziv et al (5) . Wu et al (8) however used the same formula as us in their study in kidney transplant patients, but not in the immediate post-operative period. The formula they used was $\% \mathrm{CV}=$ standard deviation of mean $/$ mean $\times 100 \%$ with a cut off value of $22.5 \%$.

The causes for this significant variation in drug handling profiles are due to the patient's changing physiology after transplant. Early in the post-transplant course, fluctuations in tacrolimus levels may result from fluid shifts, gastroparesis due to diabetic nephropathy, change in drug dosing initiated by physicians in an attempt to ensure appropriate immunosuppression and drug interactions (e.g., changes in prednisone dosing and antifungal medication). Anecdotally, until 2013 when we changed from azole antifungals to an echinocandin (micafungin), we were having to use a lower concentration of tacrolimus. Borra et al. (6) assessed the effect of high within-patient variability in tacrolimus and MMF clearance on the risk for developing the composite end point of graft loss, biopsy-proven chronic allograft nephropathy, and doubling in serum creatinine concentrations. His study
Table 2 - Cox proportional hazards analysis on the influence of covariates related to graft survival

\begin{tabular}{lcc}
\hline & $\begin{array}{c}\text { Hazard ratio } \\
\text { (95\% confidence interval) }\end{array}$ & $\boldsymbol{p}$ value \\
\hline Cold ischaemia time (CIT) & $1.263(1.040,1.534)$ & $\mathbf{0 . 0 1 9}$ \\
\hline Donor age & $1.049(0.993 .1 .108)$ & 0.086 \\
\hline Recipient age & $0.991(0.918,1.069)$ & 0.808 \\
\hline Number of HLA mismatches & $1.289(0.834,1.991)$ & 0.253 \\
\hline Episode of in patient rejection & $3.656(1.008,13.269)$ & $\mathbf{0 . 0 4 9}$ \\
\hline Tac \%CoV & $1.021(1.002,1.041)$ & $\mathbf{0 . 0 3 4}$ \\
\hline
\end{tabular}

showed that patients with higher variability in tacrolimus clearance demonstrated inferior graft outcomes despite comparable mean drug levels.

The obvious strategy to try and reduce the TacCoV $\%$ and improve patient outcomes post transplantation would be to change to a modified release version of tacrolimus, there is some data in the general transplant literature to suggest a beneficial impact (8-10)- but another more recent study did not find the switch to have an impact on intra-patient variability of Tacrolimus (11).

There are some weaknesses in our study namely its small sample size and due to its retrospective nature of our analysis we cannot provide data such as donorspecific antibodies (DSA) to distinguish between an immunological or non-immunological pathogenesis. However, on univariate analysis we identified 3 factors as being predictors of a worse graft outcome namely cold ischaemia time, an episode of rejection whilst an inpatient and a high tacrolimus variability.

\section{CONCLUSIONS}

Our study does seem to suggest that a high intrapatient tacrolimus variability, very early following a PancTx may be a predictor of graft survival-but the exact mechanisms involved are beyond the scope of the current data. Tac \%CoV is much greater in pancreas transplant recipients when compared with kidney transplant recipients. Even in the modern era with Campath induction, it is possible that more grafts are being lost to rejection related complications than we had previously realised.

Further studies regarding the variability of different tacrolimus formulations in pancreas transplant recipients are mandatory in order to avoid serious complications in this group of patients, such as acute rejection and graft loss. 


\section{Authors' contributions}

Mihnea loan lonescu collected, analysed the data and is the main author of the manuscript.

A Sewpaul, R Figueiredo and J Chmelo helped with data collection and analysis.

$\mathrm{CH}$ Wilson was the leading senior author who came up with the idea of looking into tacrolimus variability in pancreas transplant and designed the study.

A Barlow, S White and D Manas helped in reviewing the data analysis and reviewing the manuscript.

\section{Conflicts of interest and source of funding}

The authors state there were no financial, consultant, institutional and other relationships that might lead to bias or to a conflict of interest regarding this study.

\section{REFERENCES}

1. Kahan BD, Keown P, Levy GA, Johnston A. Therapeutic drug monitoring of immunosuppressant drugs in clinical practice. Clinical therapeutics. 2002;24(3):330-50; discussion 29.

2. van Gelder T. Within-patient variability in immunosuppressive drug exposure as a predictor for poor outcome after transplantation. Kidney international. 2014;85(6):1267-8.

3. O'Regan JA, Canney M, Connaughton DM, O'Kelly P, Williams Y, Collier $\mathrm{G}$, et al. Tacrolimus trough-level variability predicts long-term allograft survival following kidney transplantation. Journal of nephrology. 2015.
4. Christina S, Annunziato RA, Schiano TD, Anand R, Vaidya S, Chuang $\mathrm{K}$, et al. Medication level variability index predicts rejection, possibly due to nonadherence, in adult liver transplant recipients. Liver transplantation : official publication of the American Association for the Study of Liver Diseases and the International Liver Transplantation Society. 2014;20(10):1168-77.

5. Pollock-Barziv SM, Finkelstein Y, Manlhiot C, Dipchand Al, Hebert D, $\mathrm{Ng}$ VL, et al. Variability in tacrolimus blood levels increases the risk of late rejection and graft loss after solid organ transplantation in older children. Pediatric transplantation. 2010;14(8):968-75.

6. Borra LCP, Roodnat JI, Kal JA, Mathot RAA, Weimar W, van Gelder T. High within-patient variability in the clearance of tacrolimus is a risk factor for poor long-term outcome after kidney transplantation. Nephrology Dialysis Transplantation. 2010;25(8):2757-63.

7. Sapir-Pichhadze R, Wang Y, Famure 0, Li Y, Kim SJ. Time-dependent variability in tacrolimus trough blood levels is a risk factor for late kidney transplant failure. Kidney international. 2014;85(6):1404-11.

8. Wu MJ, Cheng CY, Chen $\mathrm{CH}$, Wu WP, Cheng CH, Yu DM, et al. Lower variability of tacrolimus trough concentration after conversion from prograf to advagraf in stable kidney transplant recipients. Transplantation. 2011;92(6):648-52.

9. Stifft F, Stolk LM, Undre N, van Hooff JP, Christiaans MH. Lower variability in 24-hour exposure during once-daily compared to twice-daily tacrolimus formulation in kidney transplantation. Transplantation. 2014;97(7):775-80.

10. Considine A, Tredger JM, Heneghan M, Agarwal K, Samyn M, Heaton ND, et al. Performance of modified-release tacrolimus after conversion in liver transplant patients indicates potentially favorable outcomes in selected cohorts. Liver transplantation : official publication of the American Association for the Study of Liver Diseases and the International Liver Transplantation Society. 2015;21(1):29-37.

11. Shuker N, Cadogan M, van Gelder T, Roodnat JI, Kho MM, Weimar W, et al. Conversion from twice-daily to once-daily tacrolimus does not reduce intrapatient variability in tacrolimus exposure. Therapeutic drug monitoring. 2015;37(2):262-9. 